\title{
Development of Virtual Instrument System for Large-scale Transport Aircraft Simulator Based on HLA
}

\author{
Ren-xia Ou ${ }^{1, a}$, Hua-lei Zhang ${ }^{2, b}$, Xu-yang Huo ${ }^{1, c}$ and Hong-bin Chen ${ }^{1, d}$ \\ ${ }^{1}$ School of Biomedical Engineering, Jinlin Medical College, Jilin 132013, China; \\ 2 The 93057 Troops of Chinese People's Liberation Army, Jilin 132102, China. \\ a52083926@qq.com, b172321768@qq.com, chuoxuyang@yahoo.cn, d820394212@qq.com
}

Keywords: High Level Architecture, GL Studio, Virtual Instrument, Multinomial Fitting.

\begin{abstract}
Aimed at the character of the numerous instruments and the high price in large-scale aircraft cabin, a half-entity emulation method of using virtual instrument with actual panel is presented. According to the basic active status and performance parameter of certain engine, a multinomial fitting method is used to establish the mathematical model of the stable state rotational speed system of the engine. The development of a typical instrument is expounded. The controlling methods of LED display and nonlinear scale are presented.
\end{abstract}

\section{Introduction}

High Level Architecture (HLA) is the latest development of system simulation technology and distributed interactive simulation standard which supports the reusability and the interoperability ${ }^{[1]}$. In large transport simulator based on HLA, virtual instrument, which is the core and foundation of virtual reality simulation, provides vivid cockpit environment for flight personnel and the operational command staff. Virtual instrument inheriting the advantages of existing computer simulation technology has a high degree of flexibility, because it can simulate the change of physical parameters in the real world only by modifying the texture image and the settings of the related parameters by the software. Meanwhile, virtual instrument has good expansibility and reusability, as long as the cockpit system according to a distributed simulation agreement can be interconnected with other simulation system according to the same agreement to finish more large-scale task of the simulation.

\section{Building method of the virtual instrument system in a large cargo plane simulator}

Maintaining the Integrity of the Specifications

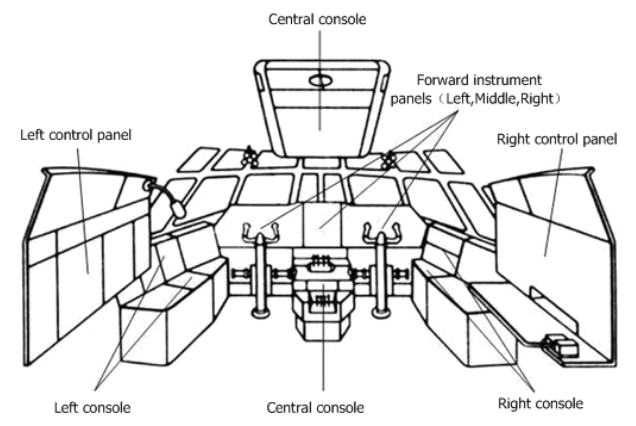

Figure 1 The panel layout of this type of cockpit instruments

The panel layout of this type of cockpit instruments and the structure of virtual instrument panels are shown in Figure 1 and Figure 2. The internal structure of this type of aircraft cockpit is complex, the numbers of instruments and the electric switches are large and physical simulation is difficult, all which can be seen from the cockpit chart. In order to reduce the development cycle and the expenses, the scheme of hardware-in-the-loop half a virtual and $\mathrm{VC}++6.0+\mathrm{GL}$ Studio3.0 which is used to develop virtual instrument are adopted in the simulator layout. Virtual instrument is displayed on the LCD panel where the physical panel including electric switches and knobs is mounted to realize 
human-machine interaction. Main controlling computer gains position signals from the helms, accelerographs, knobs and switches to control the virtual instrument by control program.

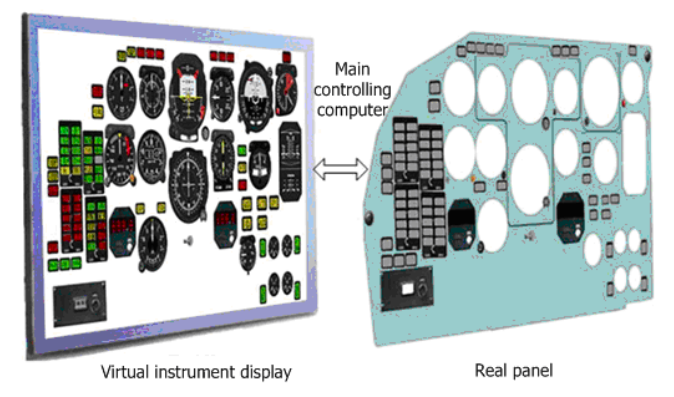

Figure 2 the structure of virtual instrument panels

\section{Mathematical model of monitoring instrument}

Virtual instrument should have the dynamic and the static performance index of the real instrument to meet demand of the virtual instrument system. The function relation between the input and output (indicating value) of the virtual instrument should be consistent with a real instrument, so the mathematical model of monitoring instrument needs to be set up. Take an engine speed instrument for an example to discus the setting process of mathematical model of monitoring instrument.

\section{Ground steady speed}

Think engine works on the ground under standard atmospheric conditions. Steady speed of the engine only has the relationship with the accelerograph location, specific corresponding relation graph as shown in Figure 3.

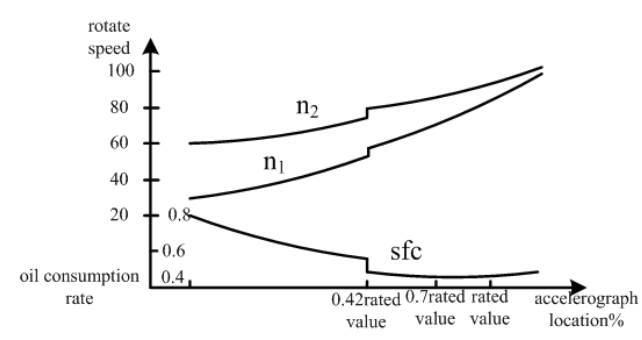

Figure 3 The relationship between steady speed, accelerograph location and oil consumption rate under standard atmospheric conditions

As shown in Figure 3, when the accelerograph is located in 0.42 of the rated value, the speed will be up to $81 \%$, air bleed valve will be opened and there will be a leap between speed and oil consumption rate. Therefore, rotation speed and oil consumption rate can be roughly divided into two stages from the low speed to the maximum.

Take the speed of $n_{2}$ for an example when air bleed valve is opened, this paper establishes the relationship between the throttle position and the speed by Lagrange's interpolation, based on the main parameters of the engine with the standard atmospheric conditions, mathematical model is as following ${ }^{[2]}$ :

$$
\begin{aligned}
& N_{2}=\frac{\left(\delta-\delta_{2}\right)\left(\delta-\delta_{3}\right)\left(\delta-\delta_{4}\right)\left(\delta-\delta_{5}\right)\left(\delta-\delta_{6}\right)}{\left(\delta_{1}-\delta_{2}\right)\left(\delta_{1}-\delta_{3}\right)\left(\delta_{1}-\delta_{4}\right)\left(\delta_{1}-\delta_{5}\right)\left(\delta_{1}-\delta_{6}\right)} n_{1}+\frac{\left(\delta-\delta_{1}\right)\left(\delta-\delta_{3}\right)\left(\delta-\delta_{4}\right)\left(\delta-\delta_{5}\right)\left(\delta-\delta_{6}\right)}{\left(\delta_{2}-\delta_{1}\right)\left(\delta_{2}-\delta_{3}\right)\left(\delta_{2}-\delta_{4}\right)\left(\delta_{2}-\delta_{5}\right)\left(\delta_{2}-\delta_{6}\right)} n_{2} \\
& +\frac{\left(\delta-\delta_{1}\right)\left(\delta-\delta_{2}\right)\left(\delta-\delta_{4}\right)\left(\delta-\delta_{5}\right)\left(\delta-\delta_{6}\right)}{\left(\delta_{3}-\delta_{1}\right)\left(\delta_{3}-\delta_{2}\right)\left(\delta_{3}-\delta_{4}\right)\left(\delta_{3}-\delta_{5}\right)\left(\delta_{3}-\delta_{6}\right)} n_{3}+\frac{\left(\delta-\delta_{1}\right)\left(\delta-\delta_{2}\right)\left(\delta-\delta_{3}\right)\left(\delta-\delta_{5}\right)\left(\delta-\delta_{6}\right)}{\left(\delta_{4}-\delta_{1}\right)\left(\delta_{4}-\delta_{2}\right)\left(\delta_{4}-\delta_{3}\right)\left(\delta_{4}-\delta_{5}\right)\left(\delta_{4}-\delta_{6}\right)} n_{4} \\
& +\frac{\left(\delta-\delta_{1}\right)\left(\delta-\delta_{2}\right)\left(\delta-\delta_{3}\right)\left(\delta-\delta_{4}\right)\left(\delta-\delta_{6}\right)}{\left(\delta_{5}-\delta_{1}\right)\left(\delta_{5}-\delta_{2}\right)\left(\delta_{5}-\delta_{3}\right)\left(\delta_{5}-\delta_{4}\right)\left(\delta_{5}-\delta_{6}\right)} n_{5}+\frac{\left(\delta-\delta_{1}\right)\left(\delta-\delta_{2}\right)\left(\delta-\delta_{3}\right)\left(\delta-\delta_{4}\right)\left(\delta-\delta_{5}\right)}{\left(\delta_{6}-\delta_{1}\right)\left(\delta_{6}-\delta_{2}\right)\left(\delta_{6}-\delta_{3}\right)\left(\delta_{6}-\delta_{4}\right)\left(\delta_{6}-\delta_{5}\right)} n_{6}
\end{aligned}
$$

In this mathematics model, $\delta$ stands for throttle position, $\delta_{1} \sim \delta_{6}$ stands for throttle positions of the six state, $N_{2}$ is on behalf of the speed of high pressure rotor, and $n_{1} \sim n_{6}$ represent six states of the high pressure rotor speed. Similarly, the relationship model between other parameters and throttle position can be established with the same method.

\section{Ground acceleration speed}

Acceleration should be checked when the engine is ground-run, throttle lever will be pushed from the local train position to push off state in 1 2 seconds, the time that the engine will take to be taking off state should keep $8 \sim 10$ seconds. The rules of revolving speed change in the process of accelerated 
speed are shown in Fig4: the process of revolving speed increased from $60 \%$ to $100 \%$ can be divided into three stages. The left extension cord of $\mathrm{CD}$, which is a straight line and the vertical axis cross at $\mathrm{e}$ point, at which the speed is $86 \%$,the total times that the engine takes from the starting rotation speed up to the largest if the starting rotation speed in the range of $60 \% \sim 86 \%$ are always equal. The rules of the simplified change to the revolving speed in the accelerating process is shown in Figure5: The rules of the revolving speed change could be described as the broken line and its turning point is $\mathrm{f}$ when the starting rotation speed is in the range of $60 \% \sim 86 \%$. The rules of the revolving speed change are that the straight $l_{3}$ is parallel to $l_{4}$ when the starting rotation speed is over $86 \%$. Suppose that the starting rotation speed is $\mathrm{N}$ and the time for acceleration is $t_{a}$, the mathematical model in the process of accelerated rotation speed is:

$$
\begin{aligned}
& l_{2}: N_{2}=4.67 t_{a}+N \quad N<N_{0}, \quad 60 \leq N<86 \\
& l_{3}: N_{2}=1.4 t_{a}+86 \quad N \geq N_{0}, \quad 60 \leq N<86 \\
& l_{4}: N_{2}=1.4 t_{a}+N \quad 86 \leq N \leq 100
\end{aligned}
$$

Mathematical expressions of turning time $\mathrm{t}_{0}$ and turning speed $\mathrm{N}_{0}$ are:

$$
t_{0}=\frac{86-N}{3.27} \quad N_{0}=122.82-0.43 N
$$

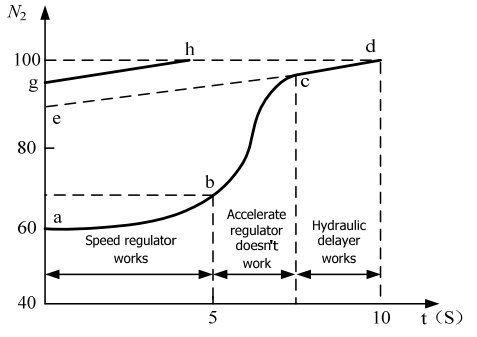

Figure 4 The rules of revolving speed change in the process of accelerated speed

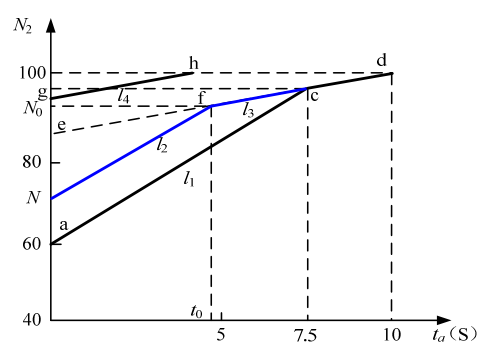

Figure 5 The rules of the simplified change to the revolving speed in the accelerating process

Similarly, the mathematical model in the engine deceleration process can be built with this method. The process of engine test can be simulated by the above-mentioned mathematical model and the simulation results are as shown in Figure 6. The simulation accuracy can meet the practical requirements.

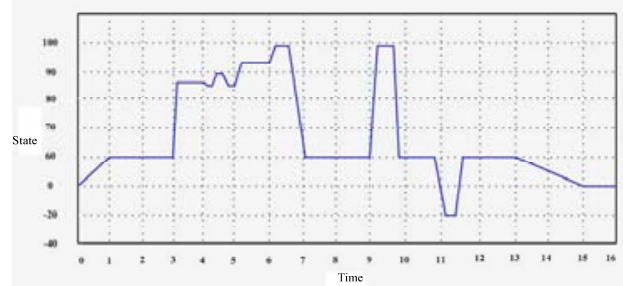

Figure 6 simulation results of the engine test

\section{Development of the typical virtual instrument}

\section{The development of the bar instrument}

Bar instrument is a metering device that digital sign and scale are pointed on the outer surface of the cylinder. Now take the instructions pilot for an example to expound the specific modeling process and the specific steps are:

a) Change photos into instrument textures according the function requirements of instructions pilot.

b) Create a polygon in the edit window then stick corresponding texture after named. A column should be created when building pitching scale, then add scale texture on this column to make the whole cylindrical surface be surrounded by scale texture ${ }^{[3]}$. Pitching scale can be displayed through the twirl of the column. 
c) Create control instructions ZLJSYClass, then generate codes in the code generators and add all the codes into the VC project.

d) Control the pitch Angle. Piecewise functions are adopted to realize the angle conversion in this paper.

void ZLJSYClass::Pitch (const float \& value)

$\{$ pitch=value;

if $\left(\right.$ pitch $>=0 \& \& \_$pitch $\left.<5\right)$

kd->DynamicRotate(_pitch*(8.4/5.0), X_AXIS);

$/ / 0 \sim 5^{\circ}$

if $\left(\right.$ pitch $>=5 \& \& \_$pitch $\left.<40\right)$

$\mathrm{kd}->$ DynamicRotate $\left((\right.$ pitch-5.0)*(64.2-8.4)/35.0+5.0, X_AXIS $) ; \quad \quad / / 5 \sim 40^{\circ}$

if $\left(\_\right.$pitch $>=40 \& \& \_$pitch $\left.<=80\right)$

kd->DynamicRotate $(($ pitch-40.0)*(135.5-8.4)/30, X_AXIS);

$\left./ / 40 \sim 80^{\circ} \quad\right\}$

e) Control the displays of the alarm signal brand and signal light, create double polygon in the edit window, paste display and no display texture respectively, at last control display by the API functions.

\section{The development of the LED display instrument development}

The LED display instrument, which is used in the modern transport, is a meter that can display numbers. Now take speed-and-drift indicator for an example to discuss the modeling processes of the LED display instrument, the specific development processes are as following:

a) The modeling processes of the instrument are the same as before. LED display model needs to be defined seven lines, named Line1 Line 7 and to be given the corresponding texture, then display numbers by the display lines. LED display model is shown in Figure 7:

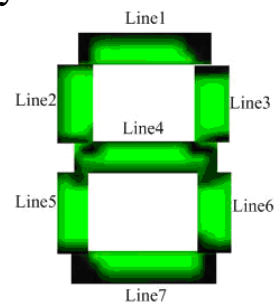

Figure 7 LED display model

b) Control the LED display. The realizing method is that controlling the seven lines to display or not to simulate the numbers that need to be displayed.

c) Digital display need to carry and therefore take the digit in the unit position or in the tens place or in the hundreds place to be assigned to the LED display module.

\section{Summary}

Based on the software platform of the Studio GL and HLA standards, a virtual instrument system in the large transport simulator is developed in this paper. An actual panel, in which the knobs, switch and LCD display are comprised, is used to simulate cockpit instruments. The development of the virtual instrument controlling model and the typical instrument are focused on. The engineering practice shows that this simulation system works well and has good reliability. At the same time, it can reduce development costs greatly. A good engineering application prospect of this simulation system is expected.

\section{References}

[1] DISTI, Inc. GL Studio Programming Guide (version 3.0)[M]. USA: DISTI, Inc. 2002: 221-226

[2] Chen Shu-jin. The Development of Virtual Instrument of Transport Plane Simulator[J]. Computer information. 2008, 22(1):264-265

[3] Liu Dong-xin. The Development of Virtual Instrument in Virtual Cockpit[J]. Computer and Modernization. 2009, 6(2):112-116 